\title{
Full Lambek Calculus with contraction is undecidable ${ }^{\star}$
}

\author{
Karel Chvalovský and Rostislav Horčík \\ Institute of Computer Science, Academy of Sciences of the Czech Republic \\ Pod Vodárenskou věží 2, 18207 Prague 8, Czech Republic \\ $\{$ chvalovsky, horcik\}@cs.cas.cz
}

Among propositional substructural logics, these obtained from Gentzen's sequent calculus for intuitionistic logic $(\mathbf{L} \mathbf{J})$ by removing a subset of the rules contraction (c), exchange (e), left weakening (i), and right weakening (o) play a prominent role, e.g. in [3] such logics are called basic substructural logics. If all above mentioned rules are removed from $\mathbf{L J}$ then the full Lambek calculus is obtained.

The decidability of such logics, i.e. their sets of theorems, usually follows from the fact that they have a cut-free sequent system. Such an argument, used in [8], however, fails if the rule of contraction is involved since the proof-search tree is then infinite. Nevertheless, already Gentzen proved $[4,5]$ that $\mathbf{L J}$ is decidable and the same was shown [7] for FL with the rules of exchange and contraction $\left(\mathbf{F L}_{\mathbf{e c}}\right)$ using an idea by Kripke [9]. It remained open whether same holds for FL with contraction $\left(\mathbf{F L}_{\mathbf{c}}\right)$ and $\mathbf{F L}$ with contraction and right weakening $\left(\mathbf{F L}_{\mathbf{c o}}\right)$. We show that these logics are, on the contrary, undecidable by showing that their common positive fragment $\left(\mathbf{F L}_{\mathbf{c}}^{+}\right)$is already undecidable.

In fact, we show that the equational theory of square-increasing residuated lattices $\left(\mathcal{R} \mathcal{L}_{c}\right)$, which are sound and complete algebraic semantics for $\mathbf{F L}_{\mathbf{c}}^{+}$, is undecidable. However, the algebraic notions were used only for convenience, the whole construction can be shown using, e.g. proof-theoretical notions, because the main ideas remain the very same.

Theorem 1. The equational theory of $\mathcal{R} \mathcal{L}_{c}$ is undecidable. Consequently, the sets of formulae provable in $\mathbf{F L}_{\mathbf{c}}^{+}, \mathbf{F L}_{\mathbf{c}}$, and $\mathbf{F L}_{\mathbf{c o}}$ are undecidable.

Note that this is not very common among known substructural logics. The undecidability of the positive fragment of the involutive distrubutive $\mathbf{F} \mathbf{L}_{\mathbf{e c}}$ is proved in [10] and the same for the equational theory of modular lattices is shown in [2].

In what follows, we give the main ideas of the proof. It was proved in [6] that the deducibility problem for $\mathbf{F L}_{\mathbf{c}}^{+}$is undecidable using a string rewriting system (SRS) which simulates Minsky machines by square-free words, i.e. the rule of contraction cannot affect them.

\footnotetext{
* The work was supported by the grant P202/11/1632 of the Czech Science Foundation and the long-term strategic development financing of the Institute of Computer Science (RVO:67985807). This abstract is based on the paper of the same title that is accepted for publication.
} 
This SRS is then equivalently expressed in terms of atomic conditional SRSs which differ from SRSs in two aspects. First, only rules with atomic right side are allowed, i.e. $x \rightsquigarrow a$ where $x \in \Sigma^{*}$ and $a \in \Sigma$. Second, the usage of every rule is restricted by a specific context in which it is applicable.

Finally, an encoding of atomic conditional SRSs in $\mathcal{R} \mathcal{L}_{c}$ is shown. Roughly speaking the conditionality in rules is expressed by join and an auxiliary rewriting system (inspired by [1]), the rewriting symbol $\rightsquigarrow$ is encoded by an implication and a set of rules by a meet of encoded rules. Although the constant 1 plays also an important role in this encoding, it can be shown that it is not necessary. Therefore even the fragments of $\mathcal{R} \mathcal{L}_{c}$ and $\mathbf{F L}_{\mathbf{c}}^{+}$containing only join, meet, and an implication are undecidable.

We conclude with some notes. The whole construction can be easily modified for logics having a weaker form of contraction $x^{k} \leq x^{l}, 1 \leq k<l$. More interestingly, as the construction, in fact, provides a chain of explicit reductions, it is possible to obtain a form of "algorithmic" deduction theorem.

Theorem 2. Let $T \cup\{\varphi\}$ be a finite set of formulae. There is an explicit algorithm that produces a formula $\psi$ (given an input $\varphi$ and $T$ ) such that $\psi$ is provable in $\mathbf{F L}_{\mathbf{c}}^{+}$iff $\varphi$ is provable in $\mathbf{F L}_{\mathbf{c}}$ from $T$.

\section{References}

1. Chvalovský, K.: Undecidability of consequence relation in full non-associative Lambek calculus. Journal of Symbolic Logic 80(2), 567-586 (2015)

2. Freese, R.: Free modular lattices. Transactions of the AMS 261(1), 81-91 (1980)

3. Galatos, N., Jipsen, P., Kowalski, T., Ono, H.: Residuated Lattices: An Algebraic Glimpse at Substructural Logics, Studies in Logic and the Foundations of Mathematics, vol. 151. Elsevier, Amsterdam (2007)

4. Gentzen, G.: Untersuchungen über das logische Schließen I. Mathematische Zeitschrift 39(1), 176-210 (1935)

5. Gentzen, G.: Untersuchungen über das logische Schließen II. Mathematische Zeitschrift 39(1), 405-431 (1935)

6. Horčík, R.: Word problem for knotted residuated lattices. Journal of Pure and Applied Algebra 219(5), 1548-1563 (2015)

7. Kiriyama, E., Ono, H.: The contraction rule and decision problems for logics without structural rules. Studia Logica 50(2), 299-319 (1991)

8. Komori, Y.: Predicate logics without the structural rules. Studia Logica 45(4), 393-404 (1986)

9. Kripke, S.A.: The problem of entailment. Journal of Symbolic Logic 24, 324 (1959), abstract

10. Urquhart, A.: The undecidability of entailment and relevant implication. Journal of Symbolic Logic 49(4), 1059-1073 (1984) 